\title{
Perfil Sociodemográfico e Hábitos de Vida de Idosos Portadores de Hipertensão
}

\section{Sociodemographic Profile and Lifestyle Habits of Elderlies with Hypertension}

\author{
VANESSA TEIXEIRA DE LIMA OLIVEIRA ${ }^{1}$ \\ EDNEYDE SAMARA SALÚSTIO DOS SANTOS ${ }^{2}$ \\ NADJA NAJARA DE ALMEIDA MEDEIROS² \\ AMOYSAARAÚJO RIBEIRO ${ }^{3}$ \\ MARIA TEREZA GOUVEIA PESSOA ${ }^{3}$
}

\section{RESUMO}

Objetivo: Rrealizar a caracterização socioeconômica e identificar o consumo alimentar de idosos portadores de hipertensão atendidos em unidades básicas de saúde de um município do Rio Grande do Norte. Materiais e métodos: Tratase de um estudo transversal, descritivo e quantitativo. A amostra $(n=160)$, de ambos os sexos, cadastrados no HIPERDIA das unidades básicas de saúde. Aplicou-se um formulário abordando dados socioeconômicos, clínicos, hábitos cotidianos e o questionário de frequência do consumo alimentar. Resultados: Observou-se $79 \%(\mathrm{n}=126)$ dos idosos do sexo feminino e faixa etária entre $60-70$ anos. A renda foi de até 1 salário mínimo e escolaridade limitada ao ensino fundamental incompleto. Quanto aos hábitos cotidianos, verificou-se que $89 \%$ não referiram tabagismo, $95 \%$ não consumiam bebida alcoólica e $53 \%$ não praticavam de atividade física. Além da hipertensão, $36 \%$ dos idosos apresentaram Diabetes Mellitus. Quanto ao consumo alimentar, $53 \%$ e $23 \%$ dos idosos consumiam temperos e molhos prontos, respectivamente. Quanto ao consumo de carnes curadas, $92 \%$, consumiam de 1 a 4 vezes por semana. Quanto aos embutidos, destaca-se o consumo de linguiça $(24 \%)$, mortadela $(23 \%)$ e salsicha $(17 \%)$. Dentre os enlatados, destaca-se o consumo de milho verde $(32 \%)$ e os derivados do leite, observou-se um considerável consumo (49\%) de queijo coalho. Conclusão: Verificou-se um maior número de idosos ainda na fase inicial do envelhecimento, com um nível sócioeconômico que os caracteriza como uma população vulnerável, que apresenta um consumo alimentar rico em cloreto de sódio, o que pode comprometer o quadro existente de hipertensão, favorecendo o surgimento de ouras doenças cardíacas.

\section{DESCRITORES}

Hipertensão. Idoso. Consumo de alimentos.

\begin{abstract}
Objective: To characterize the socioeconomic profile and identify the food intake of elderlies with hypertension treated at primary care units of a municipality in Rio Grande do Norte state. Material and Methods: A cross-sectional, descriptive and quantitative study was carried out. The sample $(n=160)$ consisted of both males and females, registered at the HIPERDIA program at the primary care units. We applied a form addressing socioeconomic and clinical data and daily habits as well as a food frequency questionnaire. Results: We observed that $79 \%$ $(n=126)$ of the elderlies were females aged 60 to 70 years, With income up to 1 minimum wage and education limited to incomplete primary education. Regarding daily habits, $89 \%$ of them did not report smoking habits, $95 \%$ did not consume alcohol, and $53 \%$ did not practice physical activity. In addition to hypertension, $36 \%$ of the elderlies also had diabetes mellitus. As to food intake, $53 \%$ and $23 \%$ of them reported consuming seasoning and ready-made sauces, respectively. A total of $92 \%$ of respondents reported the habit of consuming cured meats 1 to 4 times per week, as well as sausage $(24 \%)$, mortadella $(23 \%)$ and hot dog sausage $(17 \%)$. Among the canned food, there was consumption of green canned corn $(32 \%)$ and milk derivatives; there was a considerable consumption (49\%) of rennet cheese. Conclusion: A high number of elderlies at the very early stage of the aging process was found, with a socioeconomic level that characterizes them as a vulnerable population with a rich dietary intake of sodium chloride, which may compromise the current hypertension profile, thereby favoring the emergence of other heart diseases.
\end{abstract}

\section{DESCRIPTORS}

Hypertension. Elderly. Food Consumption.

1 Professora do Curso de Nutrição - Coordenadora da Pesquisa - Faculdade de Ciências da Saúde do Trairi/Universidade Federal do Rio Grande do Norte - Natal/RN - Brasil.

2 Nutricionista - Pesquisadora Externa - Natal/RN - Brasil.

3 Discente do Curso de Graduação em Nutrição - Bolsista de iniciação científica - Faculdade de Ciências da Saúde do Trairi/Universidade Federal do Rio Grande do Norte - Natal/RN - Brasil. 
$\mathrm{O}$ envelhecimento populacional é uma ocorrência mundial, e no Brasil este tem ocorrido de madeira rápida. As estimativas apontam que, em 2020, o Brasil possuirá um número superior a 30 milhões de pessoas, sendo desta forma o sexto país do mundo em número de idosos ${ }^{1}$. Dentro do próprio grupo etário está aumentando a proporção de idosos com 80 anos ou mais, bem como os centenários, modificando sua composição ${ }^{2}$.

A velocidade do processo de transição demográûca, ou seja, a redução na taxa de mortalidade e aumento da expectativa de vida, influencia na transição epidemiológica vivida pelo país nas últimas décadas, sendo assim, o aumento de doenças que acometem os idosos tem como principal fator de risco o próprio envelhecimento. As doenças cardiovasculares são as mais frequentes causas de mortalidade entre os idosos ${ }^{3}$.

A hipertensão arterial sistêmica (HAS) tem alta prevalência e é considerada como importante problema de saúde pública tendo relação com todas as doenças cardiovasculares e sendo avaliada como principal fator de risco para a mortalidade em todo o mundo ${ }^{4}$.

Vários fatores podem interferir na decorrência da hipertensão, estes podem ser fatores metabólicos e não metabólicos, sendo os não metabólicos fatores que surgem a partir da modificação do estilo de vida da população $0^{5}$. Fatores como, genética, idade, sexo, etnia, tabagismo, alcoolismo, fatores socioeconômicos e dietéticos, obesidade, estilo de vida sedentária, são considerados fatores de risco pertinentes ao desenvolvimento da hipertensão ${ }^{6}$. A identificação destes fatores de risco contribui para o desenvolvimento de ações de controle para esta doença.

A modificação dos hábitos cotidianos paralelo à prevenção de fatores de risco auxiliam no controle das doenças crônicas e, até no conhecimento de sua prevalência ${ }^{7}$.Justificando-se, assim, a importância da avaliação da dieta consumida, uma vez que esta é considerada um dos principais determinantes das doenças crônicas e metabólicas.

A avaliação da dieta pode ser realizada por meio de vários métodos. O questionário de frequência do consumo alimentar (QFCA) é baseado na análise da frequência usual de alimentos contidos em uma lista, por um período de tempo específico e previamente construída pelo pesquisador ${ }^{8}$. Atualmente, esse inquérito tem sido avaliado com base em grupo de alimentos9. Desta forma a lista de alimentos do questionário de frequência pode ser reduzida o que o torna mais favorável na identificação de padrões alimentares ${ }^{8}$.

Com base nos conhecimentos do perfil sóciodemográfico, condições clínicas, hábitos de vida e consumo alimentar de idosos hipertensos, são direcionadas intervenções e ações eficazes para o controle da hipertensão arterial.

O objetivo do estudo foi realizar a caracterização socioeconômica, clínica e identificar hábitos relacionados ao consumo alimentar de idosos portadores de hipertensão atendidos em unidades básicas de saúde de um município do Rio Grande do Norte.

\section{MATERIAIS E MÉTODOS}

Trata-se de um estudo com delineamento transversal, de natureza descritiva, com uma abordagem quantitativa.

O estudo realizou-se com indivíduos $(\mathrm{n}=160)$ com idade igual ou superior a 60 anos, de ambos os sexos, cadastrados no programa HIPERDIA das Unidades Básicas de Saúde da zona urbana do Município de Santa Cruz/RN. O programa HIPERDIA, do Governo Federal, é um sistema de cadastramento e acompanhamento de hipertensos e diabéticos em todas as unidades básicas do Sistema Único de Saúde e ainda garante aos cadastrados no programa, os medicamentos prescritos pelo médico.

A pesquisa foi parte de um projeto maior intitulado "Identificação de fatores de risco para hipertensão arterial e avaliação do consumo alimentar em idosos no Município de Santa Cruz-RN e este obteve o parecer favorável do Comitê de Ética em Pesquisa da Faculdade de Ciências da Saúde do Trairí - FACISA/ UFRN (CAAE: 14444213.5.0000.5568 - No parecer/ 352.839).

Foi identificado o quantitativo de idosos cadastrados no programa HIPERDIA das unidades básicas de saúde da zona urbana do município de Santa Cruz/RN. A amostra $(n=160)$ foi calculada tomando por base um erro amostral de $5 \%$ e um nível de confiança de $95 \%$.

Os dados foram coletados durante as consultas agendadas no programa, nos meses de junho a setembro de 2014. Inicialmente foram explicitados os preceitos éticos necessários à participação no estudo, atendendo as diretrizes nacionais para pesquisa com seres humanos especificadas pelo Conselho Nacional de Saúde na Resolução $N^{\circ}$ 466, de 12 de dezembro de $2012^{10}$. Após explicação, os indivíduos, assinaram ou colocaram a impressão digital, conforme condição do idoso, um Termo de Consentimento Livre e Esclarecido (TCLE).

Os participantes da pesquisa foram abordados de maneira individual, tendo sido realizada entrevista 
aplicando um formulário estruturado constituído de três etapas. Na primeira etapa foram obtidas informações sobre os dados socioeconômicos e condições clínicas dos indivíduos (sexo, idade, escolaridade, renda familiar e presença de diabetes). Na segunda etapa, foi investigado a presença de hábitos cotidianos como tabagismo, consumo de bebida alcoólica, prática e frequência de atividade física. Na terceira etapa foi aplicado o questionário de frequência do consumo alimentar. Este foi elaborado com base em um estudo piloto realizado previamente com a mesma população de idosos da região ${ }^{11}$. E foi utilizado para identificar o consumo dos alimentos ricos em cloreto de sódio, como embutidos, enlatados e derivados do leite, que fazem parte do cotidiano destes indivíduos.

Para tabulação e análise dos dados foi aplicado o software Excel 2007, para identificação da distribuição percentual das variáveis.

\section{RESULTADOS}

A Tabela 1 apresenta o perfil dos usuários segundo dados sociodemográficos, clínicos e hábitos cotidianos.

O gráfico 1 apresenta a frequência de utilização cotidiana de produtos ricos em cloreto de sódio.

A tabela 2 expõe a frequência do consumo alimentar diário dos idosos quanto ao grupo de alimentos (embutidos, carnes curadas, enlatados, bebidas e derivados do leite).

\section{DISCUSSÃO}

Do total de entrevistados, houve predomínio do sexo feminino, o que corrobora com outras pesquisas de caráter populacional ${ }^{12}$, e está compatível com a tendência de feminilização no processo de envelhecimento. Em outro estudo sobre avaliação do consumo alimentar de um grupo de idosos hipertensos do bairro Bela Vista de São José/SC, também houve predominância de mulheres $(86,6 \%)$. A maior parte da população idosa no Brasil é composta por mulheres com $55,7 \%{ }^{13}$. Além do fato da maior prevalência de mulheres na população idosa no Brasil, pode ainda ser destacado a busca mais frequente, por parte das mulheres, pelos serviços de saúde.

Considerando a faixa etária predominante neste estudo, observa-se que em uma outra pesquisa com idosos hipertensos obteve resultado semelhante ${ }^{14}$, corroborando com esses dados. Este fato pode se dar devido a que nessa faixa etária ainda se observa predomínio da capacidade funcional associado à maior independência, por isso a procura com mais frequência às unidades básicas de saúde. Além disso, pode ser um local onde esses idosos busquem a socialização, principalmente quando as unidades possuem grupos que se reúnem periodicamente.

A maioria dos idosos apresentou renda de até um salário mínimo e a escolaridade foi em sua maioria limitado ao ensino fundamental incompleto. Em outros estudos com idosos tem sido identificado o baixo poder aquisitivo assim como o baixo nível de escolaridade ${ }^{15}$. Esses baixos índices podem interferir diretamente ao acesso as informações, procura pelos serviços de saúde e a adequação ao tratamento eficaz da pressão arterial ${ }^{15}$. Pode-se, ainda, afirmar que essa baixa escolaridade, possivelmente, compromete à procura por uma terapia nutricional que seja coadjuvante à terapia medicamentosa.

Quanto à presença de alguns hábitos cotidianos, que podem influenciar diretamente no controle da hipertensão arterial, foi visto que um baixo percentual dos entrevistados autorreferiram tabagismo e ingestão de bebida alcoólica. Pesquisa realizada com adultos e idosos na zona urbana de Uberaba-MG obteve um valor superior ao encontrado nesta pesquisa, onde $19,4 \%$ tinham o hábito de fumar ${ }^{16}$. Quanto à utilização do fumo, estudos indicam que o tabagismo atual ou anterior aumenta em 36\% a chance de hipertensão arterial referida em idosas ${ }^{17}$. $\mathrm{O}$ fato de serem idosos do interior do estado que guardam hábitos mais pacatos em relação ao tabagismo e ingestão de bebidas alcoólicas, pode justificar os resultados deste estudo. A baixa prevalência de idosos que autorreferiram fumar neste estudo, pode indicar um fato positivo no tratamento e controle da hipertensão arterial.

Quanto ao consumo do álcool, há evidências que os níveis da pressão arterial podem se elevar com o consumo de bebidas alcoólicas. Portanto, a redução do seu consumo é uma das principais medidas orientadas no tratamento não medicamentoso da hipertensão ${ }^{18,19}$, sendo desta forma, de grande importância o envolvimento dos profissionais de saúde no tratamento da hipertensão, principalmente entre os idosos, com ações de promoção e prevenção à saúde para que haja mudanças no estilo de vida para qualidade de vida entre os idosos.

Do total da amostra, mais da metade dos indivíduos relataram não praticar atividade física. Um estudo realizado pela Universidade Federal de Goiás $\mathrm{GO},{ }^{20}$ o qual contou com a participação de 45 idosos portadores de hipertensão arterial em tratamento não farmacológico, evidenciou que a maioria (80\%) dos idosos pesquisados obtiveram resultado significativo 
Tabela 1 - Perfil dos usuários segundo dados socioeconômicos e hábitos cotidianos de idosos $(n=160)$ atendidos nas unidades básicas de saúde dos bairros centrais do Município de Santa Cruz-RN, 2014.

\section{Variável \\ Sexo}

Feminino

Masculino

Idade (anos)

$60-70$

$71-80$

$>80$

Escolaridade

Analfabetismo

Ensino fundamental incompleto

Ensino fundamental completo

Ensino médio incompleto

Ensino médio completo

Ensino Superior

\section{Renda Familiar}

Não informou

1 Salário mínimo

$>1$ Salário mínimo

$<1$ Salário mínimo

\section{Portador de Diabetes}

Sim

Não

\section{Tabaqismo}

Sim

Não

Consumo de bebida alcoólica

Sim

Não

$8 \quad 5$

$152 \quad 95$

Prática de atividade física

Sim

Não

$\begin{array}{ll}76 & 47\end{array}$

Frequência de atividade

física

Não tem frequência

2x Semana

3x Semana

4x Semana

$5 x$ Semana

$\begin{array}{cc}1 & 1 \\ 91 & 57 \\ 59 & 36 \\ 9 & 6\end{array}$

$\begin{array}{cc}58 & 36 \\ 102 & 64\end{array}$

$18 \quad 11$

$142 \quad 89$

$84 \quad 53$

$\begin{array}{cc}9 & 12 \\ 15 & 20 \\ 24 & 31 \\ 1 & 1 \\ 27 & 36\end{array}$




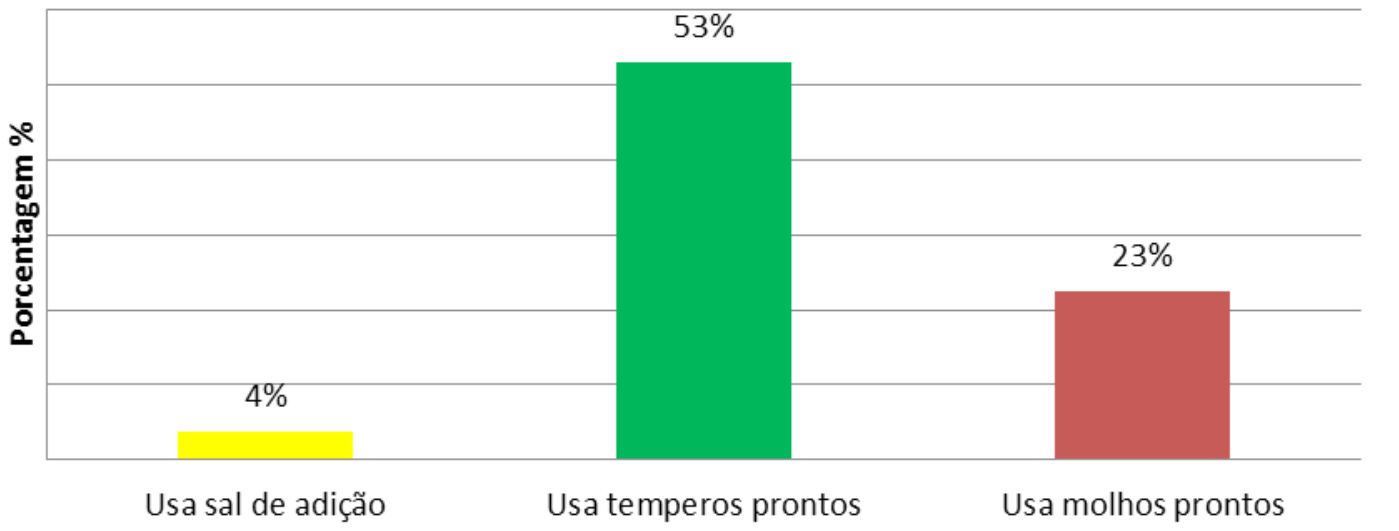

Gráfico 1 - Frequência de utilização de produtos ricos em cloreto de sódio por idosos $(n=160)$ atendidos nas unidades básicas de saúde dos bairros centrais do Município de Santa Cruz-RN, 2014.

\begin{tabular}{|c|c|c|c|c|c|}
\hline Grupo de Alimentos & Nunca & $\begin{array}{c}1 \mathrm{X} \\
\text { semana }\end{array}$ & $\begin{array}{c}2 \text { a } 4 x \\
\text { semana }\end{array}$ & $\begin{array}{l}1 \mathrm{X} \\
\text { dia }\end{array}$ & $\begin{array}{c}2 \text { ou mais } \\
x \text { dia }\end{array}$ \\
\hline & & & $\%$ & & \\
\hline \multicolumn{6}{|l|}{ Embutidos } \\
\hline Linquica & 76 & 18 & 6 & 0 & 0 \\
\hline Mortadela & 79 & 15 & 6 & 0 & 0 \\
\hline Salsicha & 83 & 12 & 5 & 0 & 0 \\
\hline Presunto & 89 & 8 & 3 & 0 & 0 \\
\hline Hambúrquer & 96 & 3 & 1 & 0 & 0 \\
\hline \multicolumn{6}{|l|}{ Carnes curadas } \\
\hline Carne de Sol & 17 & 20 & 51 & 11 & 1 \\
\hline Carne de Charque & 76 & 15 & 6 & 3 & 0 \\
\hline \multicolumn{6}{|l|}{ Enlatados } \\
\hline Milho Verde & 68 & 21 & 11 & 0 & 0 \\
\hline Ervilha & 74 & 20 & 6 & 0 & 0 \\
\hline Creme de Leite & 71 & 18 & 9 & 1 & 1 \\
\hline Maionese & 92 & 4 & 4 & 0 & 0 \\
\hline Ketchup & 94 & 4 & 2 & 0 & 0 \\
\hline \multicolumn{6}{|l|}{ Bebidas } \\
\hline Sucos Industrializados & 75 & 10 & 11 & 3 & 1 \\
\hline Refrigerante & 73 & 23 & 4 & 0 & 0 \\
\hline Refriqerante Diet & 92 & 6 & 2 & 0 & 0 \\
\hline \multicolumn{6}{|l|}{ Derivados do leite } \\
\hline Manteiqa com Sal & 77 & 8 & 8 & 6 & 1 \\
\hline Marqarina com Sal & 62 & 12 & 16 & 7 & 3 \\
\hline Queiio Mussarela & 71 & 11 & 14 & 3 & 1 \\
\hline Queiio Prato & 93 & 3 & 3 & 1 & 0 \\
\hline Queiio Manteiga & 71 & 23 & 4 & 1 & 1 \\
\hline Queiio Coalho & 44 & 20 & 29 & 5 & 2 \\
\hline
\end{tabular}


na redução da pressão arterial, com a prática de atividade física. O que comprova que os programas de atividade física pode ser um importante meio de tratamento não farmacológico para a hipertensão. Os resultados encontrados nesta pesquisa podem estar associados, ao fato de não ter nas unidades básicas de saúde um educador físico, nem tampouco programas que utilizem a atividade física como ferramenta no tratamento da hipertensão arterial. Além disso, a própria estrutura da cidade não favorece essa prática, pois possui apenas uma pista de caminhada em um dos bairros da cidade, não sendo de fácil acesso para os idosos que moram nos bairros mais distantes.

Quando analisados os dados clínicos dos idosos, pode-se observar que além da hipertensão arterial uma grande parcela dos idosos estudados também apresentam Diabetes Mellitus. Em estudo realizado em um Centro de Saúde da Família da Secretaria Executiva Regional VI de Fortaleza - CE constatou que (49,8\%) dos idosos tinham hipertensão arterial, e (62,1\%) tinham hipertensão associada ao diabetes ${ }^{21}$. O risco de apresentar diabetes é três vezes maior nos indivíduos hipertensos ${ }^{22}$. Como a hipertensão está associada a um maior grau de resistência à insulina, e os medicamentos anti-hipertensivos podem agravar este quadro, o indivíduo portador de hipertensão torna-se mais suscetível a desenvolver diabetes ${ }^{23,24}$. Tendo em vista as pesquisas clínicas realizadas, que comprovam a relação entre hipertensão e diabetes, logo o resultado encontrado nessa pesquisa já era esperado.

Quanto aos dados referentes ao consumo alimentar, no Brasil, o consumo de sal é alto, excedendo os limites máximos recomendados para a sua ingestão em todas as regiões do país e em todos os estratos de renda $^{25}$. O Plano de Ação para Enfrentamento das Doenças Crônicas Não Transmissíveis, destaca como meta estabelecida para ser atingida pelo país até 2022, redução do consumo médio diário de sal de 12 gramas (2010) para 5 gramas $(2022)^{26}$.Nesse estudo, apesar da pequena frequência de pessoas adicionarem sal à refeição, observou-se uma elevada prevalência do uso de temperos e molhos prontos, tendo em vista que tais temperos são mais ricos em sódio, do que propriamente em cloreto de sódio ${ }^{27}$. A restrição de sal tem efeito antihipertensivo, ${ }^{28}$ especialmente se combinada a outros hábitos alimentares saudáveis, e pode levar à redução da dose da medicação empregada para reduzir a pressão $\operatorname{arterial}^{29}$.

Com relação ao consumo de alimentos ricos em cloreto de sódio, o estudo identificou que dentre os grupos de alimentos mais consumidos diariamente se encontram as carnes curadas, como carne de sol e carne de charque. Num estudo realizado para avaliar o perfil antropométrico e alimentar de idosos frequentadores do Programa Universidade Aberta à Terceira Idade (UNATI), 16,13\% dos idosos consumiam diariamente carne bovina $^{30}$. Avaliando os hábitos regionais, podese perceber que o consumo dessas carnes no interior do estado do Rio Grande do Norte/RN, é um hábito comum, especialmente na região do Trairí e Seridó. Isso porque há alguns anos atrás, no interior do estado, consumir a carne de sol era a forma mais comum, uma vez que as carnes bovinas frescas eram mais difíceis de serem encontradas nos supermercados.

Ainda observou-se uma prevalência no consumo dos embutidos, enlatados, bem como de sucos industrializados. Esse consumo é um hábito mundial, sendo muito comum na região pela facilidade de aquisição nos pequenos comércios distribuídos pela cidade. Por tudo isso, observa-se a necessidade de se fazer trabalhos de conscientização com a população sobre a utilização de produtos frescos e, em alguns casos, in natura, apesar dos supermercados serem restritos e a aquisição desses produtos frescos serem de mais difícil acesso. Os produtos embutidos possuem grandes quantidades de gordura saturada, colesterol, conservantes e sódio. Sendo assim, são prejudiciais à saúde quando consumidos frequentemente. Estes podem aumentar o risco de doenças cardiovasculares, hipertensão arterial e obesidade ${ }^{30}$. Cerca de $75 \%$ do excesso sódio consumido pela população Brasileira é proveniente dos produtos industrializados ${ }^{30}$.

Com relação aos derivados do leite, vê-se frequência considerável no consumo do queijo tipo coalho, queijo mussarela e margarina com sal. Apesar de haver frequência de uma a quatro vezes na semana no consumo desses alimentos, pode-se observar que grande parte da população estudada já tem instrução sobre os problemas que o consumo exagerado destes alimentos pode causar principalmente para os hipertensos. O consumo do queijo de coalho é um hábito regional, e muitos idosos ainda preferem consumir esse queijo aos queijos brancos, acreditando que estes são "fracos" já que apresentam aspectos sensoriais diferenciados e que, por muitas vezes, não agradam.

O Guia Alimentar para a população Brasileira de 2006 recomenda o consumo diário de leite e derivados e diversos estudos associam esses alimentos a um padrão de consumo alimentar saudável ${ }^{30}$. Porém, os mesmos devem ser consumidos preferencialmente desnatados ou com baixo teor de lipídios, já que são ricos em gordura saturada ${ }^{30}$. O Guia Alimentar para a 
População Brasileira 2014, diz que alimentos de origem animal são boas fontes de proteínas e da maioria das vitaminas e minerais de que necessitamos, mas não contêm fibras e podem apresentar elevada quantidade de calorias por grama e teor excessivo de gorduras não saudáveis (chamadas gorduras saturadas), características que podem favorecer o risco de obesidade, de doenças do coração e de outras doenças crônicas ${ }^{29}$.

A terapia nutricional associada à medicamentosa torna-se ainda mais difícil por eles já terem essa cultura firme entre seus costumes no dia a dia. Isso torna o trabalho de educação nutricional importante, podendo esta ser aplicada através de ações que implementem hábitos nutricionais saudáveis, o que provavelmente, irá melhorar os costumes alimentares e, consequentemente, aumentar a qualidade de vida dessa população.

\section{REFERÊNCIAS}

1. Carvalho JAM, Garcia RA. O envelhecimento da população brasileira: um enfoque demográfico. Cad Saúde Publica. 2003;19(3):725-33.

2. Camarano AA. Envelhecimento da população brasileira: uma contribuição demográfica. Rio de Janeiro: Instituto de Pesquisa Econômica Aplicada; 2002.

3. Schmidt MS, Duncan BB, Silva GA, Menezes AM, Monteiro $\mathrm{CA}$, Barreto SM et al. Doenças crônicas não transmissíveis no Brasil: carga e desafios atuais. The Lancet.2011:61-74.

4. World Health Organization. Global health risks:mortality and burden of disease attributableto selected major risks. Geneva: WHO; 2009[acesso em 10 mar 2015].

5. Amado TCF, Arruda IKG. Hipertensão arterial no idoso e fatores de risco associados. Rev. Bras. Nutr. Clin. 2004; 19(2):94-9.

6. VI Diretrizes Brasileiras de Hipertensão Arterial. Arq Bras Cardiol. 2010;95(1 supl1):1-51.

7. Teodósio MR, Freitas CLC, Santos NRV, Oliveira ECM. Hipertensão na mulher: estudo em mães de escolares de Jaboatão dos Guararapes - Pernambuco - Brasil. Rev Assoc Med Bras. 2004; 50(2): 158-62.

8. Machado FCS, Henn RL, Olinto MTA, Anjos LA, Wahrlich V, Waissmann W.Reprodutibilidade e validade de um questionário de frequência alimentar por grupos de alimentos, em adultos da Região Metropolitana de Porto Alegre, Brasil. Rev. Nutr. 2012; 25(1):65-77.

9. Sasaki S, Kobayaski M, Tsugane S. Validity of a selfadministered food frequency questionnaire used in the 5-year follow-up survey of the JPHC Study Cohort I: comparision with dietary records for food groups. J Epidemiol. 2003; 13(1):51-6.

\section{CONCLUSÃO}

O estudo permitiu observar uma prevalência de idosos ainda na fase inicial do envelhecimento, com um nível sócioeconômico que os caracteriza como uma população vulnerável que apresenta um consumo de alimentos ricos em cloreto de sódio, o que pode comprometer o quadro existente de hipertensão arterial, favorecendo o surgimento de ouras doenças cardíacas. O perfil identificado da clientela favorece a importância da terapia nutricional como uma ferramenta importante e eficaz para o controle da hipertensão e redução da incidência de outras patologias associadas. Além de reforçar a necessidade de ações na saúde pública que gerem mudanças significativas no estilo de vida desses indivíduos, promovendo educação, saúde e consequente controle de doenças crônicas. Elevando a qualidade de vida do público alvo.

10. Brasil. Ministério da Saúde. Conselho Nacional de Saúde. Comissão Nacional de Ética em Pesquisa. Resolução 466/ 2012 sobre pesquisa envolvendo seres humanos. Brasília; 2012.

11. Job MM. Hipertensão arterial em idosos: perfil de pacientes atendidos na rede básica de saúde da periferia de Santa Cruz-RN [Monografia]. Santa Cruz: Universidade Federal do Rio Grande do Norte; 2014.

12. Massa KHC. Atividade física e uso de medicamentos antihipertensivos em idosos no município de São Paulo [Dissertação]. São Paulo: Universidade de São Paulo; 2013.

13. Silva YMS, Ramos RJ, Acioly PL. Avaliação do consumo alimentar de um grupo de idosos hipertensos do bairro bela vista de São José/SC. Revista Eletrônica Estácio Saúde [Internet]. 2014 [acesso em abr de 2015]; 3(1): 64-76.

14. Romero AD, Silva MJ, Silva ARV, Freitas RWJF, Damasceno MMC. Características de uma população de idosos hipertensos atendida numa unidade de saúde da família. Rev. Rene. 2010; 11(2):72-78.

15. Pierin AMG, Gusmão JL, Carvalho LVB. A falta de adesão ao tratamento como fator de risco para hi-pertensão arterial. Hipertensão. 2004; 7(3):100-3.

16. Miranzi SSC, Ferreira FS, Iwamoto HH, Pereira GA,Miranzi MAS. Qualidade de vida em indivíduos com Diabetes Mellitus e Hipertensão acompanhados por uma Equipe de Saúde da Família. Texto \& contexto enferm. 2008; 17(4):672-9

17. Oliveira SMJV, Santos JLF, Lebrão ML, Duarte YAO, Pierin AMG. Hipertensão arterial referida em mu-Iheres idosas: prevalência e fatores associados. Texto \& Contexto Enferm. 2008; 17(2):241-9. 
18. Sociedade Brasileira de Hipertensão, Sociedade Brasileira de Cardiologia, Sociedade Brasileira de Nefrologia. $5^{a}$ Diretrizes Brasileiras de Hipertensão Arterial. São Paulo; 2006.

19. Brandão AP, Brandão AA, Freitas EV, Magalhães MEC,Pozzan R. Hipertensão arterial no idoso. In: Freitas EV, Py L, Néri AC, Cançado FAX, Gorzoni ML, Rochas M. Tratado de geriatria e gerontologia. Rio de Janeiro: Guanabara Koogan; 2006. p. 459-73.

20. Barroso WKS, Jardim PCBV, Vitorino PV, Bittencourt A, Miquetichuc F. Influência da atividade física programada na pressão arterial de idosos hipertensos sob tratamento não farmacológico. Rev. Assoc. Med. Bras. 2008; 54(4): 328-33.

21. Silva DB, Souza TA, Santos CM, Jucá MM, Moreira TMM, Frota MA, et al. Associação entre hipertensão arterial e Diabetes em centro de saúde da família. RBPS. 2011. 24(1): 16-23.

22. Souza LJ, Chalita FEB, Reis AFF, Teixeira CL, Gicovate Neto $C$, Bastos A, et al. Prevalência de diabetes mellitus e fatores de risco em Campos dos Goytacazes, RJ. Arq Bras Endocrinol Metab 2003;47:69-74.

23. Sociedade Brasileira de Diabetes. Atualização brasileira sobre diabetes. Rio de Janeiro: Diagraphic;2005.

24. Faria AN, Zanella MT, Kohlman O, Ribeiro AB. Tratamento de diabetes e hipertensão no paciente obeso. Arq. Bras. Endocrinol. Metab. 2002; 46(2): 137-42.

25. Sarno F, Claro RM, Levy RB, Bandoni DH, Ferreira SRG, Monteiro CA. Estimativa de consumo de sódio pela população brasileira, 2002-2003. Rev Saúde Pública. 2009;43(2):219-225.

26. Malta DC, Júnior JBS. Plano de Ações Estratégicas para - Enfrentamento das Doenças Crônicas Não Transmissíveis no Brasil após três anos de implantação, 2011-2013. Epidemiol. Serv. Saúde. 2014; 23(3):389-395. DOI: $10.5123 / S 1679-49742014000300002$
27. Dickinson HO, Mason JM, Nicolson DJ, CampbellF, Beyer $\mathrm{FR}$, Cook JV, et al. Lifestyle interventionsto reduce raised blood pressure: a systematicreview of randomized controlled trials. J Hypertens.2006;24(2):215-33.

28. Mancia G, De Backer G, Dominiczak A, Cifkova R,Fagard R, Germano G, et al. 2007 Guidelines forthe Management of Arterial Hypertension: The TaskForce for the Management of Arterial Hypertension of the European Society of Hypertension (ESH) and theEuropean Society of Cardiology (ESC). Eur Heart J.2007;28(12):1462-536.

29. Lopes AF, Braga CP, Boliani E, Almeida FQA. Perfil antropométrico e alimentar dos participantes do Programa Universidade Aberta à Terceira Idade (UNATI) do Instituto de Biociências de Botucatu/SP . Rev. Ciênc. Ext. 2010; 6(1): 1-13.

30. Avozani P, Spinelli RB, Zemolin GP, Zanardo VPS. Avaliação da ingestão de sódio e o risco de hipertensão arterial em Adolescentes das escolas públicas de Erechim - RS. Perspectiva. 2014; 38 (141):141-150.

Correspondência

Vanessa Teixeira de Lima Oliveira

Rua Adeodato José dos Reis, 1100.

Condomínio Sun Family - Torre A - 504.

Parnamirim - Rio Grande do Norte - Brasil - 59152-820

Email: vanessatlima@uol.com.br 